\title{
PRODUÇÃO DE CONHECIMENTO SOBRE NARRATIVAS ORAIS: CONTRIBUIÇÕES PARA AS INVESTIGAÇÕES EM LINGUAGEM INFANTIL
}

\author{
Production of knowledge on oral narratives: \\ contributions to research on child language
}

Jáima Pinheiro de Oliveira ${ }^{(1)}$, Eveline Bonki (2), Tania Moron Saes Braga ${ }^{(3)}$, Ana Cândida Schier ${ }^{(4)}$

\begin{abstract}
RESUMO
Este estudo tem como objetivo indicar um panorama geral sobre a produção de conhecimento no que tange os padrões para o desenvolvimento da narrativa oral, nos últimos quatro anos por meio de uma revisão bibliográfica sistematizada, voltada para a temática de intervenção em linguagem infantil, com o uso de narrativas orais. A revisão foi realizada em uma única etapa, com critérios específicos, utilizando os seguintes descritores: narrativas, narrativa, narrativas de crianças, linguagem e desenvolvimento, linguagem oral, habilidades linguísticas, linguagem falada, linguagem infantil, narração/narração de histórias e linguagem. O período considerado foi de 2007 a 2011. Foram obtidos 900 registros, dentre os quais, nove (1\%) atenderam aos critérios estabelecidos para análise. A maioria dos estudos relacionou-se com análises psicolinguísticas. Apenas um estudo utilizou método experimental. No período considerado foram produzidos poucos estudos abordando narrativas orais infantis; a sua grande maioria levou em consideração, fundamentalmente, aspectos cognitivos e linguísticos no processo de desenvolvimento da habilidade narrativa; a grande maioria das pesquisas foi realizada com crianças que possuem algum tipo de alteração em seu desenvolvimento linguístico. Considera-se que a produção de conhecimento, em relação ao desenvolvimento de narrativas orais infantis, exige, atualmente, um olhar voltado para as intervenções que utilizem metodologia experimental. Por fim, considera-se que é necessária uma atenção maior em relação ao desenvolvimento típico da habilidade narrativa.
\end{abstract}

DESCRITORES: Psicolinguística; Desenvolvimento da Linguagem; Linguagem Infantil

(1) Fonoaudióloga; Docente do Departamento de Fonoaudiologia e credenciada junto ao Programa de Pós-graduação em Educação da Universidade Estadual do Centro Oeste UNICENTRO, Irati, Paraná, Brasil; Doutora em Educação pela Universidade Estadual Paulista - UNESP, Marília, SP, Brasil; Mestre em Educação Especial pela Universidade Federal de São Carlos - UFSCar, São Carlos, SP, Brasil; Especialista em Fonoaudiologia Educacional pelo ConseIho Federal de Fonoaudiologia - CFFa, Brasília, DF, Brasil.

(2) Acadêmica do Curso de Fonoaudiologia da Universidade Estadual do Centro Oeste - UNICENTRO, Irati, Paraná, Brasil.

(3) Psicóloga; Docente do Departamento de Educação Especial e credenciada junto ao Programa de Pós-graduação em Educação da Universidade Estadual Paulista - UNESP, Marília, SP, Brasil; Mestre e Doutora em Psicologia pela Universidade de São Paulo - USP, São Paulo, SP, Brasil.

(4) Fonoaudióloga da Secretaria Municipal de Educação de Mallet - PR; Mestranda do Programa de Pós-Graduação em Fonoaudiologia da Universidade Estadual Paulista "Júlio de Mesquita Filho" - UNESP, Marília, SP, Brasil.

Conflito de interesses: inexistente

\section{INTRODUÇÃO}

Estudiosos do desenvolvimento infantil têm se interessado, cada vez mais, pela temática da produção de histórias ou narrativas orais ${ }^{1-3}$. Essas investigações voltam-se para aspectos do desenvolvimento normal de crianças sem queixa, no que tange as habilidades de linguagem ${ }^{3}$, crianças com queixa de desenvolvimento nessas habilidades ${ }^{1,2} \mathrm{e}$ crianças com necessidades especiais ${ }^{4,5}$.

Esse conhecimento produzido, sem dúvida, tem contribuído com questões importantes, relativas às intervenções clínicas e no campo educacional, já que a habilidade de narrar, seja de maneira oral ou escrita, trata-se de uma das manifestações mais marcantes do desenvolvimento infantil. 
A definição de histórias tem origem nos modelos que especificam seus elementos constitutivos, sua organização, convenções e construções linguísticas típicas. Morrow ${ }^{6}$ apresenta um modelo de estrutura constando de cinco categorias, a saber: (a) cenário: enunciados que descrevem os contextos físico e social, nos quais a história se desenvolve; (b) tema: evento inicial que leva o personagem principal a reagir, normalmente para solucionar um problema que surge; (c) enredo: eventos ou tentativas nos quais o personagem principal se engaja, para atingir o objetivo ou a solução do problema; (d) resolução: atendimento do objetivo ou a solução do problema; e (e) sequência: analisa a ordem apresentada pelos elementos anteriores.

Spinillo e Martins ${ }^{7}$ definem o gênero narrativo com as seguintes estruturas: (a) início, com uma abertura convencional (introdução da cena: informações sobre o tempo e lugar; personagens: suas características, motivações e metas a alcançar); (b) meio (evento, trama, situação-problema); e (c) final (resolução da situação-problema) com um fechamento convencional. A história que possui essa estrutura é considerada coerente.

À medida que é desenvolvida a capacidade de narrar fatos e eventos, essa habilidade se aprimora a tal ponto que a criança passa não somente a relatar experiências vivenciadas, mas também a recontar histórias e utilizar-se dessa capacidade para criar novos cenários e personagens ${ }^{8,9}$. Segundo Koch e Elias $^{10}$, a familiaridade com essa habilidade coloca a criança em contato com um gênero textual que Ihe será apresentado posteriormente, sem que ela saiba. Quer dizer, o desempenho da criança em relação ao gênero textual narrativo advém dos modelos aos quais ela foi exposta socialmente, ao longo de seus comportamentos linguísticos ${ }^{10}$.

As habilidades narrativas escritas também tem sido objeto de investigações de diversos pesquisadores $^{11-13}$. Estes estudos têm auxiliado questões importantes do desenvolvimento da linguagem escrita, especialmente, aquelas que se referem às intervenções educacionais ${ }^{14}$.

Diante desse contexto, essa revisão tem como objetivo analisar a produção de conhecimento e as investigações que buscam padrões para o desenvolvimento da narrativa oral infantil, fornecendo um perfil desses estudos, nos últimos quatro anos. Para alcançar tal propósito, foram consideradas as pesquisas relacionadas a essa temática, com a pretensão de indicar um panorama geral sobre essas produções na área de linguagem infantil. Espera-se contribuir com o direcionamento de novas pesquisas nessa área.

\section{MÉTODO}

Trata-se de uma revisão bibliográfica sistematizada, que abordou a temática de intervenção em linguagem infantil, com o uso de narrativas orais. A revisão foi realizada em uma única etapa, com critérios específicos, na base de dados Scielo. Essa base foi escolhida, porque nela é possível identificar alguns periódicos cujo tema está relacionado ao escopo deste estudo, são eles: Revista Brasileira de Educação Especial; Psicologia: Reflexão e Crítica; Psicologia: Teoria e Pesquisa; Pró-fono Revista de Atualização Científica; Psicologia em Estudo; Revista da Sociedade Brasileira de Fonoaudiologia; Estudos de Psicologia; Psicologia Escolar e Educacional, Revista CEFAC, dentre outros.

Os termos ou descritores utilizados foram: narrativas, narrativa, narrativas de crianças, linguagem e desenvolvimento, linguagem oral, habilidades linguísticas, linguagem falada, linguagem infantil, narração/narração de histórias e linguagem.

A fim de refinar a busca e direcioná-la para os objetivos do estudo, foram estabelecidos alguns critérios. O principal deles foi o período que, deveria ser compreendido entre 2007 e 2011. O segundo critério estabelecido foi o de subtemas, ou seja, foi dada prioridade aos artigos que tratavam de intervenções e, em seguida, aqueles com foco para diagnóstico ou aspectos do desenvolvimento da linguagem oral. Como terceiro critério estabeleceu-se que as investigações deveriam envolver estudos experimentais, quase-experimentais ou delineamentos com sujeito único, sendo que todos deveriam apresentar condições experimentais nas quais houvesse manipulação de variáveis independentes (programas, métodos), seja para modificar variáveis dependentes (habilidades de fala) ou simplesmente para medi-las em situações distintas. Como quarto critério estabeleceu-se que não seriam considerados estudos com populações com necessidades especiais, haja vista, que o principal objetivo desta revisão é contribuir com aspectos descritivos para a busca de padrões para o desenvolvimento da narrativa oral. Por fim, também poderiam ser consideradas revisões sistemáticas e metanálises sobre 0 assunto.

Num primeiro momento da análise de dados, foi utilizada a análise estatística descritiva. Os dados obtidos foram sistematizados numa tabela, na qual foram indicados os seguintes aspectos: descritor utilizado na busca, frequência absoluta de estudos encontrados e frequência absoluta de estudos selecionados. Em seguida, elaborou-se um resumo descritivo de cada estudo selecionado, considerando seus aspectos principais (objetivos, métodos e resultados). Por fim, os estudos foram discutidos 
do ponto de vista da abordagem psicolinguística, de modo a indicar as principais contribuições destes estudos para futuras pesquisas acerca da temática.

\section{REVISÃO DA LITERATURA}

\section{a) Estudos registrados e selecionados}

Observa-se, na Tabela 1, que foram encontrados 900 estudos com os descritores pré-estabelecidos, na base de dados Scielo. No entanto, apenas nove $(1 \%)$ estudos atenderam aos critérios estabelecidos. Alerta-se para a ênfase dada a essa revisão: intervenções em linguagem infantil. Com isso, pode-se inferir que são muito escassas as pesquisas com tal foco. A seguir, na descrição destes estudos, ainda será possível observar que, mesmo dentro dessa frequência, somente uma pesquisa ${ }^{15}$ utilizou um delineamento do tipo experimental. Ou seja, poucas, dentre aquelas existentes, no que se refere às intervenções em linguagem infantil, fazem uso de controles metodológicos rigorosos. É preciso enfatizar que seis estudos apareceram nos resultados para buscas de mais de um descritor.

Diante disso, embora a produção de narrativas orais infantis seja um tema específico, observa-se que tem sido dispensada pouca atenção de profissionais especializados, em relação à produção de conhecimentos, acerca dessa temática. Por se tratar de uma das mais marcantes habilidades do processo de desenvolvimento, esse tratamento deve ser repensado. Além disso, trata-se de um tema que permite diferentes formas de análise, ou seja, o estudo das narrativas infantis não está atrelado a nenhum tipo especial de abordagem teórica.

Tabela 1 - Distribuição de estudos registrados e selecionados por descritores

\begin{tabular}{lcc}
\hline Descritores & $\begin{array}{c}\text { Frequência de estudos } \\
\text { registrados }\end{array}$ & $\begin{array}{c}\text { Frequência de estudos que } \\
\text { atenderam aos critérios }\end{array}$ \\
\hline Narrativas & 68 & 1 \\
Narrativa & 91 & 3 \\
Narrativas de crianças & 1 & 1 \\
Linguagem e desenvolvimento & 1 & 1 \\
Linguagem oral & 6 & 0 \\
Habilidades linguísticas & 1 & 0 \\
Linguagem falada & 1 & 0 \\
Linguagem infantil & 76 & 1 \\
Narração/narração de histórias & 17 & 2 \\
Linguagem & 638 & 6 \\
Totais parciais & 900 & 15 \\
Total de estudos selecionados & & 9 \\
\hline
\end{tabular}

\section{b) A despeito das pesquisas selecionadas}

Nesse tópico serão descritos e, posteriormente, discutidos, os nove estudos selecionados para análise.

No primeiro deles ${ }^{1}$, as autoras buscaram caracterizar a ordenação temporal de figuras e a narração de histórias, em escolares com desenvolvimento típico de linguagem (DTL) e com distúrbio específico de linguagem (DEL). Participaram da pesquisa dois grupos: um grupo controle (GP), formado por 60 crianças, que não apresentavam alteração de linguagem e um grupo de pesquisa (GP), composto de 20 participantes, com diagnóstico de distúrbio específico de linguagem (DEL). A idade dos participantes variou de sete a dez anos. No estudo foram utilizadas 15 histórias, representadas por figuras, compostas de quatro cenas. Os dados coletados foram transcritos e analisados conforme o tipo de discurso e o tipo de organização de figuras feito pelas crianças.

A coleta desse estudo foi dividida em duas etapas. A primeira analisou a organização e narração de histórias por escolares em desenvolvimento típico e a segunda teve o mesmo objetivo, porém com crianças que apresentavam DEL. Obteve-se como resultado no primeiro estudo, que a capacidade de ordenação temporal de figuras já está presente em crianças com DTL aos sete anos. Observou-se também que, com o aumento da idade, essas crianças diminuem o uso de discursos descritivos e 
aumentam o uso de discurso intencional. Pôde-se observar, ainda, que quanto maior a complexidade das sequências, maior o uso de discurso causal e intencional. Já no segundo grupo, observou-se que não houve diferença nos discursos nas faixas etárias do grupo com DEL. Comparando-se os dois grupos, as autoras concluíram que o grupo com DEL apresentou um discurso menos elaborado (descritivo), e o grupo com DTL um discurso mais elaborado (causal). As autoras ressaltaram ainda que escolares com DEL apresentaram mais dificuldades do que escolares com DTL, na utilização da língua, ou seja, nas habilidades linguísticas necessárias para a narração de uma história1.

Numa segunda pesquisa analisada, Dadalto e Goldfeld $^{3}$ objetivaram analisar aspectos constitutivos de histórias e relatos, em crianças de cinco e seis anos de idade. Participaram desse estudo 50 crianças, de ambos os sexos, sendo 23 meninos e 27 meninas, de classes de pré-alfabetização de quatro escolas particulares da cidade de Vitória, no Espírito Santo. O critério para participação no estudo era o de que não houvesse queixas familiares, nem escolares, sobre o desenvolvimento e a aprendizagem das crianças. A amostra de análise foi constituída por uma narrativa de uma história conhecida e um relato pessoal. Foi solicitado a cada criança que narrasse a história de "Chapeuzinho Vermelho", por ser uma história conhecida e, em seguida, que ela fizesse um relato de uma situação vivida.

Como resultados, a população estudada apresentou: conhecimento da estrutura da narrativa, com domínio satisfatório da relação temporal entre eventos narrados, fazendo utilização de orações que expressam a dependência temporal com verbos de ação no pretérito (perfeito e imperfeito); utilização de operadores narrativos com predominância de "aî" e "depois"; utilização de pontos de referência temporal e espacial com propriedade; uso de relações espaço-temporais organizadas e; as relações causais encontravam-se em fase de estruturação.

Ao final do estudo, as autoras puderam concluir que as crianças apresentaram maior facilidade na produção da história do que no relato. Além disso, concluíram também que respondem a todo tipo de pergunta eliciadora. Por outro lado, a maior parte das crianças precisou de mediação no relato, embora todas respeitassem a sequência temporal tanto na história quanto no relato. E por fim, as autoras enfatizaram que as crianças usaram de maneira correta as figuras na história e, como elemento de coesão causal e temporal, usaram o "aí", tanto na história como no relato ${ }^{3}$.
Souza e Sperb ${ }^{16}$ realizaram um estudo com o objetivo de comparar o desempenho narrativo de grupos de sujeitos com atraso e distúrbio fonológico e em aquisição típica de linguagem. Participaram dessa pesquisa 63 sujeitos: $32 \mathrm{com}$ atraso, 12 com distúrbio e 19 em aquisição típica. Os participantes foram avaliados e classificados fonologicamente, por meio do instrumento de Zilles e da narrativa inventada, ambas analisadas por tipologia narrativa. Aos pais e professores foram aplicados questionários abordando aspectos de interações narrativas. Os resultados indicaram diferenças estatisticamente significantes entre o grupo com distúrbios e o controle, respectivamente, com pior e melhor desempenhos. O grupo em aquisição típica demonstrou o melhor desempenho na narrativa livre. As autoras concluíram que a tipologia fonológica apresentou-se parcialmente relacionada com o desempenho narrativo.

Num quarto estudo analisado, durante a revisão, duas autoras ${ }^{2}$ realizaram uma pesquisa com o objetivo de verificar o tempo de narrativa e de pausa, os números de palavras e de intervenções do interlocutor em narrativas orais de crianças. Participaram do estudo 31 crianças, divididas em quatro grupos etários, sendo eles: GI (3:1 a 4:0 anos), Gll (4:1 a 5:0 anos), GIII (5:1 a 6:0 anos) e GIV (6:1 a 7:0 anos). Nesse estudo, todas as amostras foram coletadas em vídeos, transcritas e analisadas estatisticamente. Os resultados obtidos indicaram valores significantemente maiores, em situação de narrativa espontânea, para as variáveis de tempo de pausa e tempo de narrativa. Por outro lado, o número de palavras foi significantemente maior em situação de livro. Quanto ao número de intervenções do interlocutor, houve relação significante entre essa variável e a idade das crianças, em contexto de livro. À medida que a idade da criança aumentava, o número de intervenções do interlocutor diminuía.

Foi possível concluir que as crianças apresentaram uma narrativa mais extensa no contexto de relato com livro sem palavras, em relação ao contexto de narrativa espontânea. Porém, não houve diferenças significantes entre as idades. $O$ estudo permitiu concluir, ainda, que a participação do interlocutor faz-se menos necessária, conforme aumenta a idade do narrador.

Em um estudo etnográfico, realizado por Smith, Bordini e Sperb ${ }^{17}$, as autoras investigaram os contextos e a interlocução na construção de narrativas orais de crianças, durante a rotina escolar, numa turma de educação infantil. Participaram do estudo 14 crianças entre cinco e seis anos e seus educadores. Foram registradas as narrativas e os contextos nas quais as mesmas foram produzidas. A análise dessas produções foi realizada por meio 
da delimitação de eventos específicos e organizados, conforme a presença dos três elementos estruturais básicos da narrativa: orientação, complicação e resolução ou resultado.

Em narrativas completas, foi considerada a presença de todos estes elementos e nas narrativas incompletas, apenas dois ou um destes. A partir deste critério, as autoras classificaram as narrativas em intraconversacionais (NIs) e eliciadas (NEs), em ordem cronológica. As autoras verificaram que as crianças narraram principalmente nos momentos informais, e menos nas oportunidades de fala eliciada e apoiada pela professora. Identificaram-se 55 narrativas intraconversacionais (NIs) em momentos da rotina, nos quais a interação verbal era livre. A maior proporção de NIs aconteceu durante as atividades não dirigidas ou pouco dirigidas. Os resultados, segundo as autoras, sugeriram a necessidade de ampliar a escuta, o estímulo e a interlocução entre pares, para promover diferentes gêneros e estilos narrativos em todos os momentos da rotina escolar.

Em outra pesquisa ${ }^{15}$, cinco autoras avaliaram os efeitos de um procedimento de ensino de categorias estruturais de histórias sobre a discriminação de seus elementos definidores em novas histórias. Participaram do estudo oito crianças, sendo duas meninas e seis meninos, alunos de $1^{\underline{a}}$ e $2^{\underline{a}}$ séries do Ensino Fundamental, que tinham entre seis anos e 11 meses e oito anos e nove meses, no início da pesquisa. Foram utilizadas histórias escritas pelas autoras, planejadas de sorte a incluir conteúdos relativos a todas as categorias de histórias. Adotaram-se "fichas de avaliação de ensino" específicas para cada história e um delineamento de linha de base múltipla entre categorias, que requereu a avaliação de todas elas, antes do início da fase de ensino e após o ensino de cada categoria, por meio de testes específicos, também elaborados pelas autoras. Cada sessão de ensino era dividida em duas etapas: definição das categorias estruturais e identificação das categorias.

Os dados foram examinados com base no percentual de respostas corretas, durante a fase de ensino e no teste de identificação de categorias estruturais. Os resultados evidenciaram que o procedimento de ensino de discriminação de categorias estruturais de histórias produziu um aumento considerável nos escores de acerto na tarefa de identificação das categorias, para todos os participantes. O procedimento avaliado mostrou-se eficiente para estabelecer discriminações das categorias estruturais de histórias e para favorecer a discriminação dos elementos definidores das categorias em novas histórias. Durante a etapa de ensino, todos os participantes revelaram a aquisição das categorias estruturais de histórias, porém, para todos, foi necessária a apresentação de histórias adicionais, uma vez que não alcançaram o critério de aprendizagem nas duas primeiras histórias, conforme era previsto no estudo. As autoras concluíram que, depois da aquisição de discriminações entre as categorias explícitas nas histórias, o passo seguinte de um programa de ensino de discriminações complexas deve consistir na identificação de elementos implícitos, por meio de respostas a perguntas inferenciais sobre uma história ${ }^{15}$.

Befi-Lopes, Bento e Perissinoto ${ }^{18}$ também realizaram uma pesquisa envolvendo a narração de histórias por crianças com distúrbio específico de linguagem (DEL). O objetivo das pesquisadoras foi o de caracterizar a narração de histórias por crianças com DEL, em relação ao tipo e ao conteúdo do discurso, para fins de comparação do desempenho destes sujeitos com seus pares cronológicos, em desenvolvimento típico. Participaram do estudo dois grupos: o grupo controle (GC), sem alterações de linguagem, composto por 24 sujeitos e o grupo pesquisa (GP), composto por oito sujeitos, com diagnóstico de DEL. Para a realização do estudo foi utilizada uma série de 15 histórias, representadas por figuras, compostas por quatro cenas cada. As autoras obtiveram como resultados que as crianças com DEL apresentam narrativas mais rudimentares, se comparadas com sujeitos que possuem desenvolvimento típico de linguagem. Esse resultado, segundo as autoras, independe do tipo de história fornecida.

As autoras concluíram que independentemente do tipo de história fornecida, a dificuldade das crianças com DEL está na utilização da língua, ou seja, nas habilidades linguísticas necessárias na narração de histórias e não na percepção dos estados mentais dos personagens.

Nas pesquisas consideradas na revisão, havia somente uma revisão de literatura ${ }^{19}$, cujo objetivo foi o de analisar as produções existentes sobre o desenvolvimento da habilidade da criança para narrar suas experiências pessoais, bem como sobre o papel do suporte verbal oferecido pela mãe durante esse desenvolvimento. As autoras deram ênfase aos estudos relacionados à abordagem sociointeracionista, embora também citassem outras análises. A revisão foi desenvolvida considerando estudos da última década, com os seguintes descritores: narrativa, criança, experiências pessoais. Essa busca foi realizada na base Scielo, nos indexadores eletrônicos Google e PsychINFO. Foi considerada também a lista de periódicos da CAPES (Coordenação de Aperfeiçoamento de Pessoal de Nível Superior) com revistas de conceito (Qualis) A, B e C. As autoras consideraram, por fim, 
o cruzamento de referências mais citadas sobre narrativas de experiências pessoais de crianças, bem como estudos clássicos sobre tema.

O texto das autoras foi dividido em discussões que privilegiaram os seguintes tópicos: Uma perspectiva sociointeracionista para estudar o desenvolvimento da criança; Aprender a narrar na interação com o outro; Das narrativas conjuntas à autoria da própria história de vida; $A$ experiência e a forma narrativa e; $O$ desenvolvimento da habilidade para narrar uma experiência vivida. Após dissertarem sobre a produção de conhecimentos acerca de cada um destes subtemas, as autoras consideraram que na última década houve uma produção interessante de pesquisas brasileiras sobre narrativas de crianças, com destaque para as áreas da psicolinguística e da psicologia cognitiva. Esses estudos permitiram a análise de relações entre aspectos cognitivos e de linguagem. Os estudos indicaram uma tendência para o uso de procedimentos que solicitam à criança a criação ou recontagem de histórias, a partir de apoios visuais (livros, figuras, dentre outros), em detrimentos daqueles que valorizam as experiências pessoais. Aqui, cabe ressaltar que alguns autores que compartilham da abordagem discursiva argumentam que a narrativa, ainda que produzida com apoio, possibilita a representação simbólica da linguagem e transmite algo passado em termos de tempo e ausente em termos de espaço ${ }^{20}$.

Produzir narrativas, sejam elas orais ou escritas, envolve inúmeros conhecimentos linguísticos e cognitivos. Por isso, existem diferentes formas de abordar esse desenvolvimento. No entanto, um ponto importante e comum observado nos estudos descritos trata-se da interação. Sem dúvida, a habilidade de narrar, assim como todos os aspectos relacionados à linguagem, envolve elementos interacionais.

De modo geral, observou-se que quase todos os estudos descritos estão voltados para abordagens psicolinguísticas, isto é, com análises que privilegiam competências linguísticas e cognitivas, no processo de construção da narrativa oral ${ }^{20}$.

De modo a reforçar essa ideia, alguns autores elencam etapas no processo de aquisição e desenvolvimento da narrativa. Segundo Cavalcante e Mandrá ${ }^{2}$, o desenvolvimento da narrativa oral começa por volta dos dois anos de idade. Nessa idade, as histórias são rudimentares, pois a criança ainda não tem todos os elementos cognitivos e linguísticos adequados para organizar e estruturar uma narrativa. Por isso, pode-se dizer que ela depende da intervenção de um interlocutor para organizar os acontecimentos, contextualizando, assim, essa narrativa produzida pela criança.
Outros aspectos relacionados às habilidades narrativas também são enfatizados, em outras abordagens. No estudo de Smith, Bordini e Sperb ${ }^{17}$, por exemplo, as autoras comentaram que narrar permite à criança, a organização das dimensões lógicas que seriam a noção de tempo, de espaço, de causalidade, dentre outras. Essas noções normativas estão relacionadas às regras que devem ser seguidas para produção de uma narrativa. Isso, por sua vez, possibilitaria, também, uma compreensão das ações e intenções dos seres humanos inseridos em um mundo físico e sociocultural ${ }^{17}$. Estas autoras concordam, também, que a capacidade de narrar histórias, oralmente, passa para a narrativa escrita, de maneira gradual.

Nessa revisão não foi encontrado nenhum estudo com foco para a abordagem discursiva. Em estudos anteriores, com essa perspectiva, alguns autores ${ }^{20}$ abordaram a importância do uso de apoio visual para a construção da narrativa infantil. Nesse estudo foi comentado que a produção linguística, a partir de um apoio visual, não evidencia apenas a competência narrativa das crianças, isto é, seus movimentos do espaço discursivo. Na medida em que é oferecida à criança uma gravura, ou desenho, ela constrói, por meio da leitura da imagem, histórias entre o mundo real e imaginário, ampliando assim, os espaços da leitura e possibilitando a recriação de sentidos. Portanto, pode-se inferir que a leitura da imagem é parte da leitura de mundo e ao mesmo tempo é influenciada pelas experiências e conhecimentos prévios do sujeito leitor. Isso quer dizer que a imagem não é apenas um reflexo do mundo, mas ela remete também à história individual e social de cada leitor.

Por fim, ressalta-se que nessa revisão, a maioria dos estudos descritos trata-se de pesquisas com crianças que possuem algum tipo de alteração em seu desenvolvimento linguístico, o que indica a necessidade de produção de conhecimento, em relação ao desenvolvimento típico de narrativas orais.

\section{CONCLUSÃO}

A revisão realizada permitiu concluir que: a) no período entre 2007 e 2011 foram produzidos nove estudos abordando narrativas orais infantis, considerando 11 descritores em língua portuguesa, relacionados ao desenvolvimento de linguagem; b) a descrição dos estudos permitiu concluir que 
a sua grande maioria situa-se em estudos psicolinguísticos, que levam em consideração, fundamentalmente, aspectos cognitivos e linguísticos no processo de desenvolvimento da habilidade narrativa; c) a grande maioria das pesquisas foi realizada com crianças que possuem algum tipo de alteração em seu desenvolvimento linguístico e d) apenas um estudo usou delineamento experimental.
Estes dados permitem considerar que a produção de conhecimento, em relação ao desenvolvimento de narrativas orais infantis, exige, atualmente, um olhar voltado para as intervenções, a fim de que as contribuições sejam aplicadas ao aperfeiçoamento desse processo. Permite considerar, por fim, que é necessária uma atenção maior em relação ao desenvolvimento típico da habilidade narrativa.

\begin{abstract}
The purpose of this study is to indicate an overview about knowledge production regarding the standards for the development of oral narrative in the past four years through a systematic literature review, focused on intervention thematic in child language, with the use of oral narratives. The review was held in a single step, with specific criteria, using the following descriptors: storytelling, narrative, children narratives, language and development, oral language, linguistics skills, spoken language, child language, narration/storytelling and language. The period considered was from 2007 to 2011. There were obtained 900 records, among which, nine (1\%) met the criteria established for this review. The majority of studies were related to psycholinguistics analysis. Only one study used experimental method. In the analyzed period, few studies addressing children's oral narratives were produced; the vast majority took into consideration, primarily, cognitive and linguistic aspects in the development process of narrative skill; the vast majority of researches have been conducted with children who have any type of alteration in their linguistic development. It is believed that the production of knowledge, in relation to the development of oral narratives for children, currently requires a care towards interventions that use experimental methodology. Finally, it is believed that greater attention is required in relation to the development of narrative skills.
\end{abstract}

KEYWORDS: Psycholinguistics; Language Development; Child Language

\section{REFERÊNCIAS}

1. Bento ACP, Befi-Lopes DM. Organização e narração de histórias por escolares em desenvolvimento típico de linguagem. Pró-Fono R. Atual. Cient. [periódico na internet]. 2010 [acesso em 02 de agosto de 2011], 22(4): 503-8. Disponível em: www.scielo.br.

2. Cavalcante PA, Mandrá PP. Narrativas orais de crianças com desenvolvimento típico de linguagem. Pró-Fono R. Atual. Cient. [periódico na internet]. 2010. [acesso em 23 de março de 2011], 22(4): 391-6. Disponível em: www.scielo.br.

3. Dadalto EV, Goldfeld M. Características comum a narrativa oral de criança na pré-alfabetização. Rev. CEFAC. [periódico na internet]. 2009. [acesso em 03 de março de 2010], 11(1): 42-9. Disponível em: www.scielo.br.

4. Soares AD, Goulart BNG, Chiari BM. Competência narrativa em crianças deficientes auditivas e ouvintes: estudo transversal analítico. São Paulo Med. J. [periódico na internet]. 2010. [acesso em 11 de agosto de 2011], 128(5): 284-8. Disponível em: www.scielo.br.

5. Soares EMF, Pereira MMB, Sampaio TMM. Habilidade pragmática e Síndrome de Down. Rev. CEFAC [periódico na internet]. 2009. [acesso em 02 de agosto de 2011], 11(4): 579-86. Disponível em: www.scielo.br.

6. Morrow LM. Effects of structural guidance in story retelling on children's dictation of original stories. Journal of Reading Behavior. 1986: 18(2): 135-52.

7. Spinillo AG, Martins RA. Uma análise da produção de histórias coerentes por crianças. Psicologia: Reflexão e Crítica.1997: 10(1): 219-48.

8. Brockmeier J, Harré R. Narrativa: problemas e promessas de um paradigma alternativo. Psicologia: Reflexão e Crítica. 2003: 16(3): 525-35.

9. Shiro M. Genre and evaluation in narrative development. J. Child Lang. 2003: 30(2): 165-95.

10. Koch IV, Elias VM. Ler e escrever - estratégias de produção textual. 2 Ed. São Paulo: Contexto; 2010. 
11. Ferreira SP, Correia JA. Influência de diferentes contextos de intervenção na escrita de histórias por crianças, Estudos de Psicologia. [periódico na internet]. 2008. [acesso em 12 de outubro de 2010], 25(4): 547-55. Disponível em: www.scielo.br.

12. Cárnio MS, Alves DC, Rehem LO, Soares AJC. Práticas de narrativas escritas: atuação fonoaudiológica. Rev. CEFAC. [periódico na internet]. 2011. [acesso em 01 de julho de 2011], ahead of print. Disponível em: www.scielo.br.

13. Romano-Soares S, Soares AJC, Cárnio MS. Práticas de narrativas escritas em estudantes do ensino fundamental. Pró-Fono Revista de Atualização Científica. [periódico na internet]. 2010. [acesso em 02 de fevereiro de 2011], 22(4): 379-84. Disponível em: www.scielo.br.

14. Olinghouse NG. Student and instruction-level predictors of narrative writing in third-grade students. Reading and Writing. 2008: 21(2): 3-26.

15. Ribeiro DM, Pascoalon JF, Sella AC, Bandini CSM, Souza DG. Avaliação de um procedimento de ensino de categorias estruturais de histórias. Psicol. esc. educ. [periódico na internet]. 2009. [acesso em 23 de outubro de 2010], 13(2): 303-14. Disponível em: www.scielo.br.
16. Souza APR, Sperb CBR. Desempenho narrativo em sujeitos com distúrbio/atraso fonológico. Rev. CEFAC. [periódico na internet]. 2009. [acesso em 03 de março de 2011], 11(3): 389-95. Disponível em: www.scielo.br.

17. Smith VH, Bordini GS, Sperb TM. Contextos e parceiros do narrar de crianças na escola infantil. Psicologia: Reflexão e Crítica. [periódico na internet]. 2009. [acesso em 17 de março de 2011], 22(2): 181-90. Disponível em: www.scielo.br.

18. Befi-Lopes DM, Bento ACP, Perissinoto J. Narração de histórias por crianças com distúrbio específico de linguagem. Pró-Fono R. Atual. Cient. [periódico na internet]. 2008. [acesso em 02 de março de 2011], 20(2): 93-8. Disponível em: www. scielo.br.

19. Macedo L, Sperb TM. O desenvolvimento da habilidade da criança para narrar experiências pessoais: uma revisão da literatura. Estudos de Psicologia. [periódico na internet]. 2007. [acesso em 21 de abril de 2011], 12(3): 233-41. Disponível em: www.scielo.br.

20. Bitar ML. Produção oral de crianças a partir da leitura de imagens. 1 Ed. São Paulo: Humanitas, 2002.

http://dx.doi.org/10.1590/S1516-18462012005000108

RECEBIDO EM: 08/12/2011

ACEITO EM: 28/02/2012

Endereço para correspondência:

Jáima Pinheiro de Oliveira

Universidade Estadual do Centro-Oeste

(UNICENTRO)

Setor de Ciências da Saúde - SES/I

Departamento de Fonoaudiologia

PR 153 - km 07 - Riozinho - Caixa Postal 21

Irati - PR

CEP: 84500-000

E-mail: jaimafono@gmail.com 